\title{
V1 spinal neurons regulate the speed of vertebrate locomotor outputs
}

\author{
Simon Gosgnach ${ }^{1 \star}$, Guillermo M. Lanuza ${ }^{1 \star}$, Simon J. B. Butt ${ }^{3} \dagger$, Harald Saueressig ${ }^{1}$, Ying Zhang $^{1}$, \\ Tomoko Velasquez ${ }^{1}$, Dieter Riethmacher ${ }^{4}$, Edward M. Callaway ${ }^{2}$, Ole Kiehn ${ }^{3} \&$ Martyn Goulding
}

The neuronal networks that generate vertebrate movements such as walking and swimming are embedded in the spinal cord ${ }^{1-3}$. These networks, which are referred to as central pattern generators (CPGs), are ideal systems for determining how ensembles of neurons generate simple behavioural outputs. In spite of efforts to address the organization of the locomotor CPG in walking animals ${ }^{2,4-6}$, little is known about the identity and function of the spinal interneuron cell types that contribute to these locomotor networks. Here we use four complementary genetic approaches to directly address the function of mouse V1 neurons, a class of local circuit inhibitory interneurons that selectively express the transcription factor Engrailed1. Our results show that V1 neurons shape motor outputs during locomotion and are required for generating 'fast' motor bursting. These findings outline an important role for inhibition in regulating the frequency of the locomotor CPG rhythm, and also suggest that V1 neurons may have an evolutionarily conserved role in controlling the speed of vertebrate locomotor movements.

Several genetically defined classes of neurons have been identified in the developing spinal cord ${ }^{7}$, including a class of ipsilaterally projecting inhibitory neurons that innervate motor neurons, the Engrailed1 (En1)-expressing V1 neurons ${ }^{8-11}$. To assess the function of V1 neurons in the locomotor CPG, we used two mouse models that have selective loss of the En1-expressing V1 neuronal population (Fig. 1). This was achieved through the altered specification of V1 neurons in Pax6-knockout $\left(\mathrm{Pax6}^{-/-}\right)$mice and by the selective ablation of these neurons in En1 ${ }^{\mathrm{Cre}} ;$ R26-lac ${ }^{\text {flox }} / D T A$ (En1-DTA; ref. 12) mice. At embryonic day (E) 12.5, a marked reduction in En1-positive V1 neuron cell numbers was apparent in spinal cords isolated from $\mathrm{Pax6}^{-1-}$ and En1-DTA embryos (Fig. 1a, e). This was confirmed using an $E n 1^{\text {Cre }}$ lacZ or green fluorescent protein (GFP) reporter system ${ }^{9}$, which also showed that significantly fewer En1-derived V1 neurons are present at E18.5 (Fig. 1b, f). Both Pax6 $^{-1-}$ and En1-DTA mice had normal numbers of lumbar spinal motor neurons that were appropriately organized into lateral and medial motor columns. (Fig. 1c, g). Spinal cords from $\mathrm{Pax}^{-1-}$ mice did show an increase in commissural V0 and V3 interneuron cell numbers, coupled with a slight decrease in ipsilaterally projecting Chx10-positive V2 neurons ${ }^{13}$ (Chx10 is a marker of V2 neurons; Supplementary Fig. S1). However, there were no changes in ventral

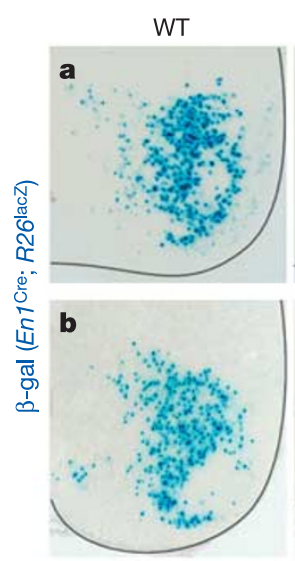

Pax6 $^{-/-}$
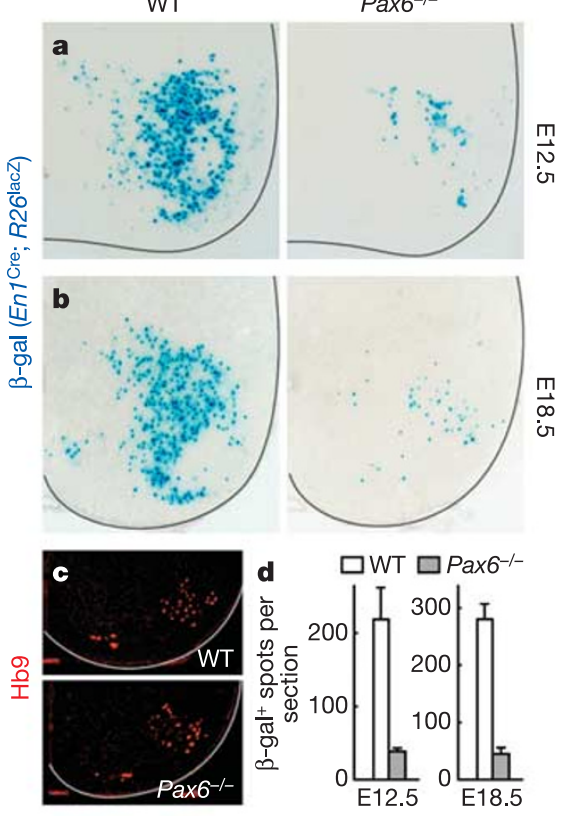
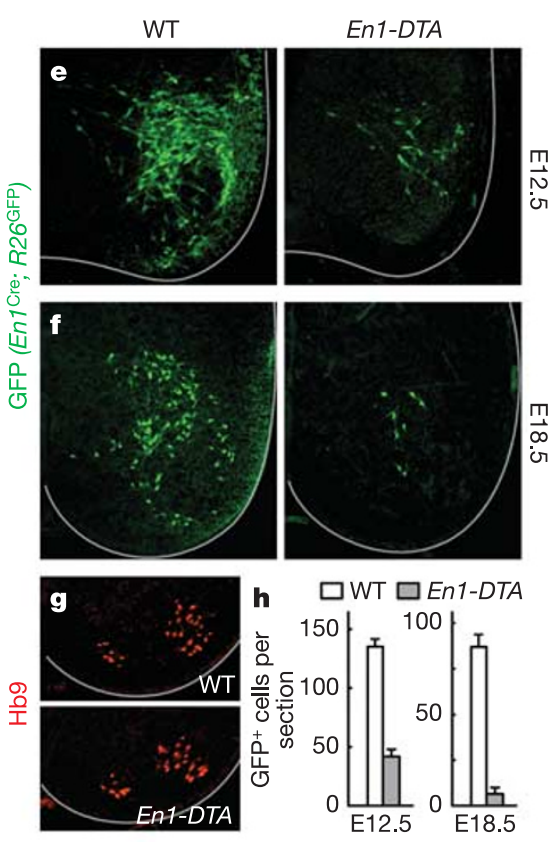

Figure $1 \mid$ V1 neurons are reduced in $\mathrm{Pax6}^{-/-}$ and En1-DTA spinal cords. a, b, Cross-sections through E12.5 (a) and E18.5 (b) wild-type (WT) and Pax6 ${ }^{-1-}$ spinal cords, showing fewer En1-positive V1 neurons in the Pax6 ${ }^{-1-}$ spinal cord. V1 neurons were marked using $E n 1^{\text {Cre }} ; R 26^{\text {lacZ }}$ reporter alleles. c, Lumbar-level $\mathrm{Hb} 9$ staining of motor neurons is largely unaffected in $\mathrm{Pax6}^{-1-}$ embryos.

d, Quantification of V1 neuron loss in the Pax $^{-/-}$spinal cord. e, f, En1-DTA embryos show a selective loss of En1-positive V1 neurons at E12.5 (e) and E18.5 (f). g, Numbers of $\mathrm{Hb} 9$-positive motor neurons and columnar organization are normal in the En1-DTA spinal cord. h, Quantification of V1 neuron loss in the En1-DTA cord. Error bars indicate s.d.

\footnotetext{
${ }^{1}$ Molecular Neurobiology Laboratory and ${ }^{2}$ System Neurobiology Laboratory, The Salk Institute for Biological Studies, 10010 North Torrey Pines Road, La Jolla, California 92037, USA. ${ }^{3}$ Mammalian Locomotor Laboratory, Department of Neuroscience, The Karolinska Institute, Retzius vag 8, Stockholm 17177, Sweden. ${ }^{4}$ Centre for Molecular Neurobiology, University of Hamburg, Hamburg 20251, Germany. †Present address: Department of Developmental Genetics, The Skirball Institute, New York University, New York, New York 10016, USA.

*These authors contributed equally to this work.
} 


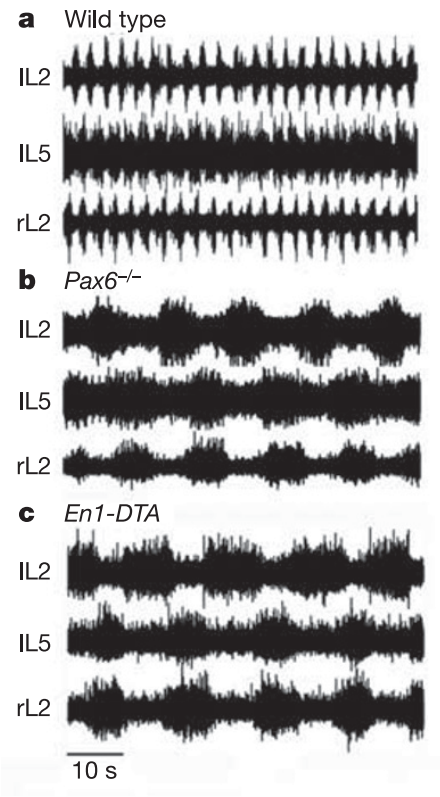

Figure 2 | Mice lacking V1 neurons show slow locomotor-like activity. a-c, Left, electroneurograms from left (lL2) and right (rL2) L2 and left L5 (IL5) ventral roots after application of $5 \mu \mathrm{M}$ NMDA and $10 \mu \mathrm{M} 5$-HT, showing locomotor-like activity in E18.5 spinal cords from wild-type (a), Pax6 $^{-1-}$ (b) and En1-DTA (c) mice. Right, circular plots showing the

interneuron specification in the En1-DTA mice (Supplementary Fig. S1).

Limbed walking movements in vertebrates are characterized by the repetitive oscillatory bursting of motor neurons, in which flexorextensor and left-right motor activities alternate. These locomotorlike oscillations can be induced in the isolated spinal cord by excitatory neurotransmitter agonists, and typically show a stepcycle period of 2-4 s (refs 5, 14, 15 and Fig. 2a). We asked whether V1 neurons regulate the coordination of flexor-extensor motor neurons during locomotion, because many V1 neurons differentiate into Renshaw cells and Ia inhibitory interneurons, two inhibitory cell types that have been proposed to coordinate flexor and extensor muscle activity around ipsilateral limb joints ${ }^{9,16-19}$. Spinal cords lacking V1 neurons showed a normal pattern of alternating flexor (L2) activity and extensor (L5) activity (Fig. 2a-c). Left-right alternation (that, is IL2 versus rL2) was also normal. However, 'locomoting' Pax6 ${ }^{-1-}$ and En1-DTA spinal cords did show a significant lengthening of both the step-cycle period and motor neuron burst duration (Fig. 2b-e).

Whereas $5 \mu \mathrm{M}$ NMDA ( $N$-methyl-D-aspartate) in combination with $5 \mu \mathrm{M}$ serotonin (5-hydroxytryptamine or 5-HT) typically induces robust locomotor-like activity in wild-type spinal cords ${ }^{15}$ (Fig. 2a), we were only able to elicit a stable pattern of locomotor-like activity in $\mathrm{Pax6}^{-1-}$ spinal cords by increasing the concentration of 5-HT to 7.5-20 $\mu \mathrm{M}$ (Fig. 2b, e). In experiments using $10 \mu \mathrm{M}$ 5-HT, the step-cycle period $(8.4 \pm 2.9 \mathrm{~s}$, mean \pm s.d. $)$ and burst duration $(4.4 \pm 1.0 \mathrm{~s})$ in $\mathrm{Pax6}^{-1-}$ spinal cords $(n=7)$ were increased significantly ( $P<0.001$, Student's $t$-test) compared to wild-type and heterozygous $\left(\mathrm{Pax6}^{+/-}\right)$spinal cords (step-cycle period $3.8 \pm 0.8 \mathrm{~s}$, burst duration $1.7 \pm 0.8 \mathrm{~s} ; n=10$ ). This relative increase in the step-cycle period was seen over a wide range of 5-HT concentrations (Fig. 2e). Similar lengthening of the step cycle (Fig. 2c, d) and burst duration was seen in En1-DTA mice $(9.3 \pm 2.3 \mathrm{~s}$ and $5.4 \pm 1.1 \mathrm{~s}$, respectively; $n=5$ spinal cords; $P<0.001, t$-test). V1 neurons are therefore dispensable for flexor-extensor coordination, but have an essential role in determining the speed of the locomotor rhythm.

Current-clamp recordings of motor neurons in wild-type and $\mathrm{Pax6}^{-1-}$ spinal cords (Fig. 3a-d) revealed that $\mathrm{Pax6}^{-/-}$motor
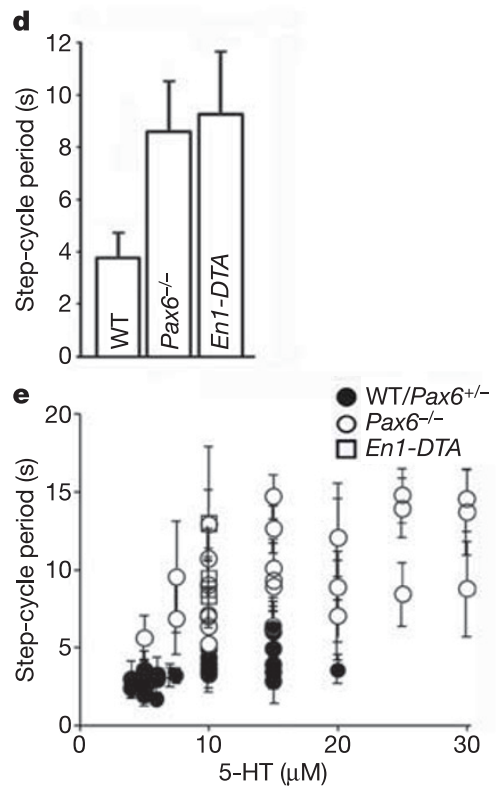

coupling of bursts in IL5 and rL2 with respect to IL2. Each point represents the vector point for one experiment. d, Mean step-cycle period in wild-type, Pax $^{-1-}$ and En1-DTA spinal cords. e, Plot of step-cycle period versus 5-HT concentration for Pax6 $6^{-I-}$ (open circles), En1-DTA (open squares) and wild-type $/ \mathrm{Pax}^{+/-}$spinal cords (filled circles). Error bars indicate s.d.

neurons show prolonged periods of membrane potential depolarization, and that they continue to fire action potentials throughout this depolarized phase (Fig. 3c). This is consistent with flexor- and extensor-related CPG neurons remaining active for longer periods of time. These intracellular recordings also revealed that the alternating excitatory and inhibitory drive that normally underlies rhythmic changes in motor neuron membrane potential ${ }^{20}$ is still present in the Pax6 $6^{-/-}$spinal cord (Fig. 3e). Importantly, Pax6 $6^{-1-}$ motor neurons still receive the phasic inhibitory inputs that are essential for coordinating alternating flexor and extensor activity (Fig. 3f). The origin of these phasic inhibitory inputs is not knownalthough they could in principle be derived from contralateral commissural interneurons ${ }^{21,22}$, this seems not to be the case, as ipsilateral locomotor coordination is normal in the $\mathrm{Paxb}^{-1-}$ cord after spinal cord hemisection (data not shown).

We then used En1 ${ }^{\text {tlZ/tlZ }}$ null 'knock-in' mice (see ref. 8) to address the function of V1 neurons in the intact animal. Spinal cords isolated from E18.5 En1 ${ }^{\text {tlZltlZ }}$ mice show a 3-4-fold slowing of the locomotor step cycle and lengthening of the burst duration (Fig. $4 \mathrm{a}, \mathrm{b}$; $11.2 \pm 2.3 \mathrm{~s}$ and $6.1 \pm 1.8 \mathrm{~s}$, respectively; $n=10$ spinal cords), which closely resemble the behavioural changes seen in $\mathrm{Pax6}^{-1-}$ and En1-DTA spinal cords (compare with Fig. 2). Using a Wnt1 ${ }^{E n 1}$ transgene ${ }^{23}$ to rescue the En1 mutant midbrain-hindbrain phenotype, we obtained adult $E n 1^{t l Z / t l Z}$ mice in which the V1 neuron connectivity defects in the spinal cords ${ }^{8,9}$ are not rescued (H.S., unpublished observation). When rotarod tests were performed on 4-6-month-old En1 ${ }^{t l Z l t l Z}$; Wnt1 ${ }^{\text {En1 }}$ mice and age-matched controls, $E n 1^{t l Z / t l Z}$; Wnt1 ${ }^{E n 1}$ mice showed a clear deficit in their ability to walk and maintain their balance at higher rotarod speeds (Fig. 4c; see Methods). Although the duration (and the speed) for which the mutant animals walked on the rotarod increased during the trial period, at no time did they attain walking speeds above 18 r.p.m. However, these animals did walk on the rotarod for extended periods of times at idling speed ( 5 r.p.m., data not shown). In contrast, wildtype animals walked and maintained their balance at much higher speeds (approximately 22 r.p.m. on day 1 and up to 35 r.p.m. on day 5). This suggests that adult En1 mutants are impaired in their ability to perform quick stepping movements. 

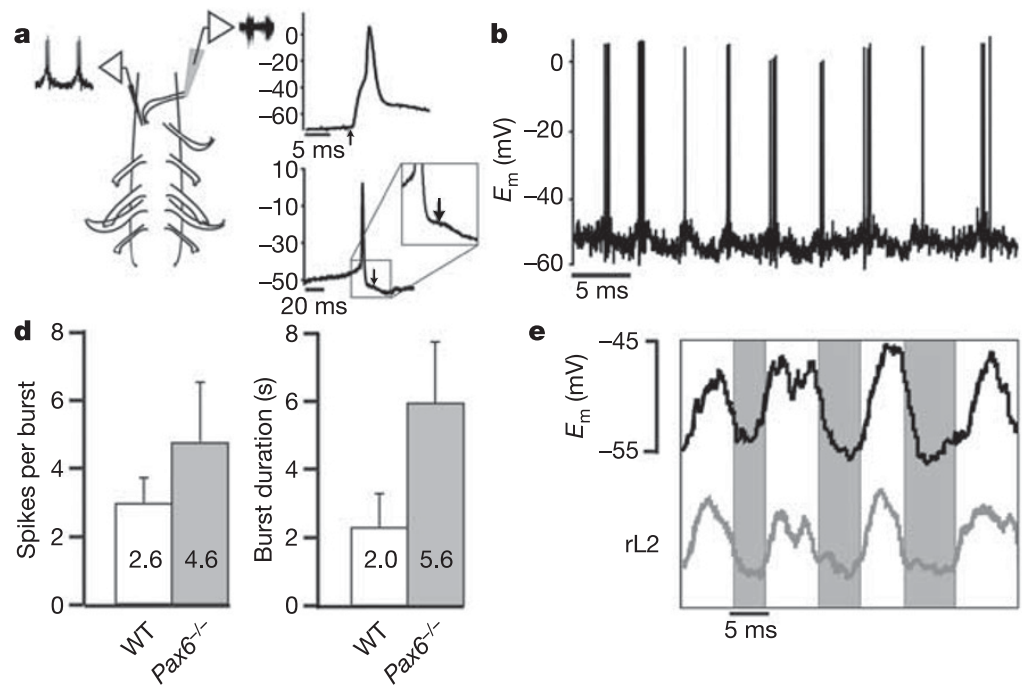

e

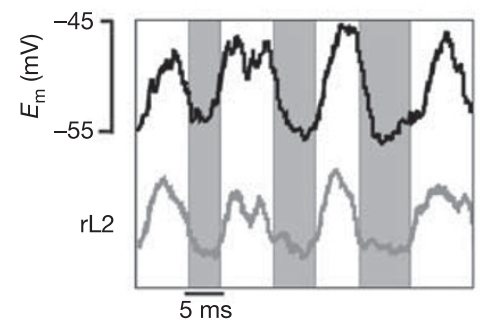

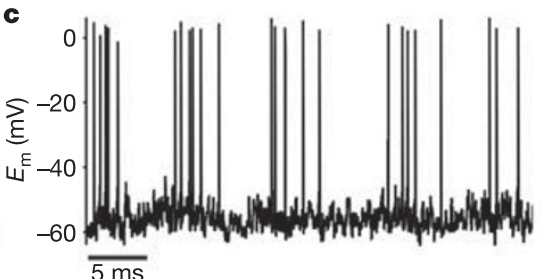

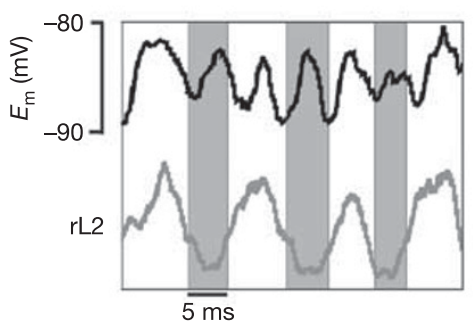

Figure 3 Motor neuron firing during locomotion in the Pax6 ${ }^{-/-}$spinal cord is prolonged and shows rhythmic inhibition. a, Schematic of recording setup, with a suction electrode recording electroneurogram activity from rL2 ventral root and an intracellular electrode recording from a motor neuron located in L2. Motor neurons were identified by the presence of an antidromic action potential in response to ventral root simulation (upper right panel, arrow) and by a small after-depolarization following orthodromic spikes (lower right panel, arrow), which is unique to rodent motor neurons. b, c, Recording from motor neurons located in rL2 of wild-type (b) and $\mathrm{Pax6}^{-1-}$ (c) mice after application of NMDA and 5-HT. d, Chart showing the mean number of action potentials (mean \pm s.d.) fired per depolarization of the motor neuron and the mean duration of the

Next, we used transgenic mice (AlstR192 mice) that conditionally express the Drosophila allatostatin G-protein-coupled receptor (AlstR) to test whether acutely suppressing V1 neuron excitability causes a similar lengthening of the locomotor step cycle (Fig. 5a). AlstR couples to endogenous mammalian GIRK channels (inwardrectifying $\mathrm{K}^{+}$channels), causing a decrease in cellular input resistance and neuronal excitability ${ }^{24}$. In control experiments, allatostatin $(100 \mathrm{nM}-5 \mu \mathrm{M})$ had no effect on motor rhythm generation, locomotor coordination or the step-cycle period (Supplementary Fig. S2a). In contrast, spinal cords from nestin ${ }^{\text {Cre }}$; AlstR192 mice showed strong allatostatin-dependent depression in rhythmic motor activity (Supplementary Fig. S2b).

En1 ${ }^{\mathrm{Cre}}$; AlstR192 mice were then used to selectively express AlstR in V1 neurons (Fig. 5a, b). In spinal cord slices prepared from En1 ${ }^{\mathrm{Cre}}$; AlstR192 mice, we observed a pattern of GFP reporter expression that was identical to the normal distribution of $\mathrm{V} 1$ neurons (Fig. 5c). Whole-cell recordings from identified GFPpositive V1 neurons ( $n=7$ cells) revealed a reversible decrease in neuronal excitability in response to current steps (Fig. 5d) and ramps (Fig. 5e) after the addition of allatostatin $(10 \mathrm{nM})$. The decrease in excitability (2-3-fold), although not as pronounced as in isolated adult neurons ${ }^{24}$, was nevertheless substantial, and it was not seen in control, AlstR-negative neurons (Supplementary Fig. S2c).

When locomotor-like activity was induced in postnatal day P0-P2 En1 ${ }^{\text {Cre }}$; AlstR192 spinal cords ( $5 \mu \mathrm{M}$ NMDA + $10 \mu \mathrm{M}$ 5-HT; Fig. $\left.5 f\right)$, we observed a step-cycle period of $3-4 \mathrm{~s}(3.3 \pm 0.8 \mathrm{~s}, n=15$ spinal cords), comparable to that seen in wild-type animals of a similar age. Application of allatostatin $(1-5 \mu \mathrm{M})$ to these spinal cords resulted in marked and significant lengthening of the step-cycle period $(7.9 \pm 1.8 \mathrm{~s}, n=15$ spinal cords; $P=0.002$, paired $t$-test), which was reversed upon washing out the neuropeptide (Fig. 5f). As such, lengthening of the locomotor step cycle that results from the defects in V1 neuron development can be reproduced when V1 neuronal activity is suppressed. Two recent studies have provided evidence that modifying neuronal activity during development can alter the depolarized phase (LDP) of the motor neuron during locomotion in wild-type and $\mathrm{Pax}^{-1-}$. e, f, Intracellular recording from a motor neuron located in the rL2 segment (upper traces) and electroneurogram recording from the $\mathrm{rL2}$ ventral root (lower traces, rectified and integrated) in a $\mathrm{Pax6} 6^{-1}$ mouse. Grey area indicate the period during which $\mathrm{rL2}$ is inactive. In e, recordings were performed at resting membrane potential $\left(E_{\mathrm{m}}-50 \mathrm{mV}\right)$ after application of $5 \mu \mathrm{M}$ NMDA $+15 \mu \mathrm{M} 5$-HT. The motor neuron is active in-phase with ventral root activity. In $\mathbf{f}$, the motor neuron is hyperpolarized to $-88 \mathrm{mV}$, beyond the calculated $\mathrm{Cl}^{-}$reversal potential, which results in the emergence of out-of-phase depolarization owing to reversed inhibitory currents.

neurotransmitter phenotype of spinal neurons ${ }^{25}$ and produce small changes in CPG motor outputs ${ }^{26}$, indicating that activity may have a role in organizing these locomotor networks However, our results show that the changes in locomotor CPG outputs following the acute
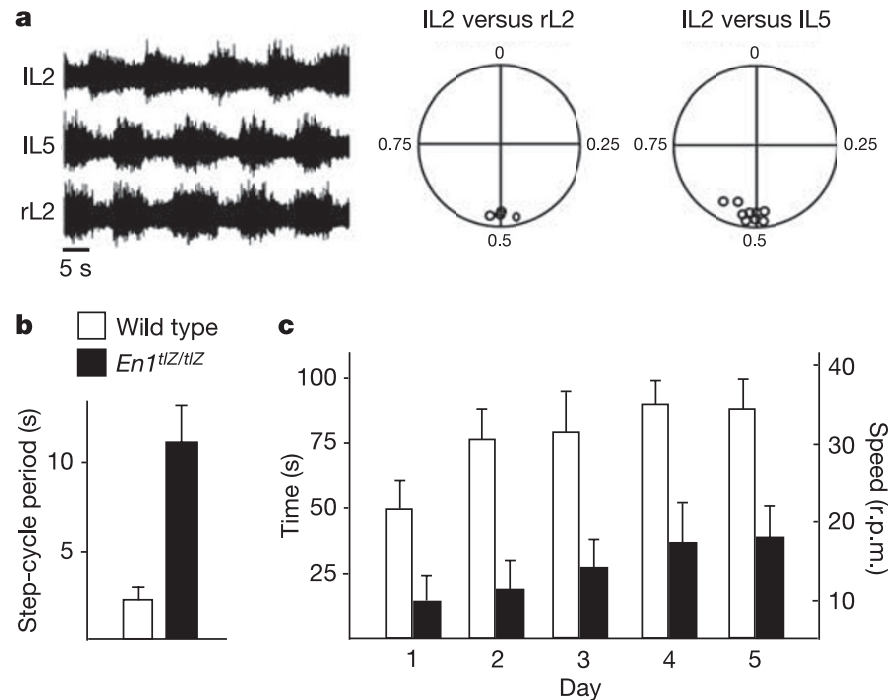

Figure 4 | En1 ${ }^{t \mid Z / t l Z}$ mice show a slowing of stepping movements. a, Left, electroneurogram recordings from IL2, IL5 and rL2 ventral roots after application of $5 \mu \mathrm{M}$ NMDA/7.5 $\mu \mathrm{M} 5$-HT to an isolated E18.5 En1 $1^{\mathrm{tlZ} / \mathrm{tlZ}}$ spinal cord. Right, circular plots indicate normal ventral root alternation. Each small circle corresponds to the vector point for a single experiment. b, Graph showing the increase in the step-cycle period in En $1^{t l Z / t l Z}$ mutants compared with wild-type spinal cords. c, Rotarod testing of mouse motor behaviours, showing the time and bar speed that age-matched wild-type (open bars) and En1 ${ }^{t l Z / t l Z}$; Wnt $1^{E n 1}$ mice (filled bars; three animals each) were able to run on an accelerating rotarod. Error bars in b, c show s.d. 
a

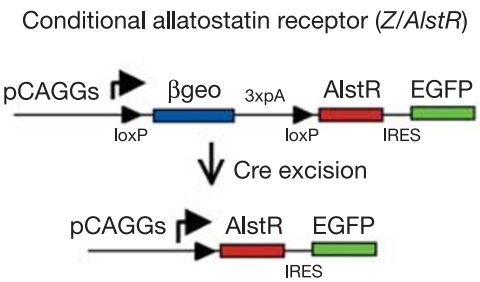

d

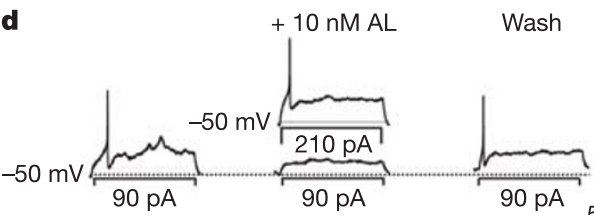

f

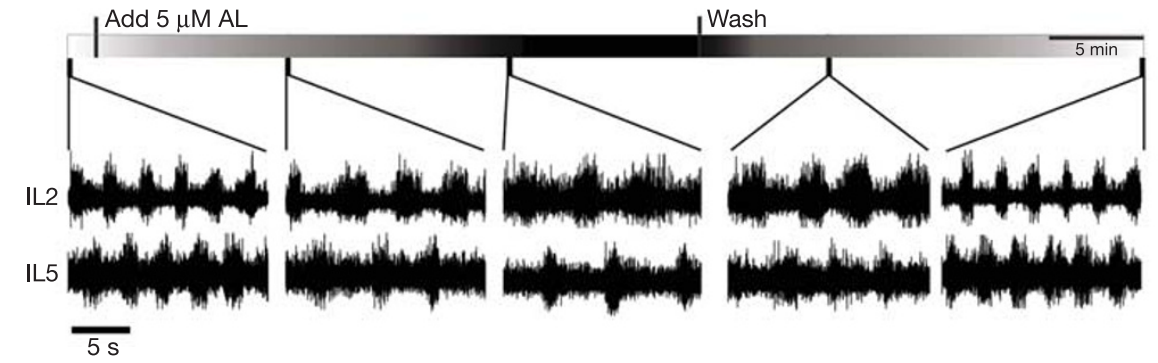

Figure 5 | Acute silencing of $\mathbf{V} 1$ neurons via the allatostatin receptor causes a slowing of locomotor-like rhythmicity. a, Schematic showing the allatostatin receptor $(Z / A l s t R)$ transgene. Crossing mice that harbour this transgene with an $E n 1^{\mathrm{Cre}}$ mouse excises the loxP-flanked $\beta$-geo sequences, leading to transcription of AlstR in En1-derived cells. $\mathbf{b}$, Co-expression of GFP (green) and En1 (red) in an E11.5 En1 ${ }^{\mathrm{Cre}}$; AlstR192 mouse, showing V1 cells expressing AlstR. Many newborn V1 neurons in the subventricular zone are AlstR/GFP-negative owing to a delay in En1-dependent Cre removal of the stop cassette. c, Spinal cord slice from a P0 En1 ${ }^{\mathrm{Cre}}$; AlstR192 mouse carrying the $Z n G$ reporter allele, showing GFP expression in V1

suppression of V1 neurons compared with the early loss of V1 neurons are highly concordant. This suggests that the absence of V1 neurons during development does not markedly reconfigure the spinal locomotor CPG network.

Our findings outline an essential role for V1 neurons in mammals in regulating the duration of the locomotor step cycle, and hence the speed of the locomotion. This function is specific for V1 neurons, as we have not seen marked changes in the frequency or duration of the step cycle when either V0 or V3 neurons are deleted from the spinal $\operatorname{cord}^{5}$ (M.G., unpublished observations). Our conclusion that V1 neurons are necessary for 'fast' locomotor outputs in mice is supported by studies in Xenopus tadpoles showing that aIN neurons, the homologues of mouse V1 neurons, are the primary source of early cycle inhibition to the swimming locomotor $\mathrm{CPG}^{10}$. Although the role of aIN neurons in regulating the speed of Xenopus swimming movements was not examined, a strong correlation was seen between aIN-derived inhibitory inputs to CPG neurons and the frequency of swimming movements ${ }^{10}$. This raises the possibility that the Xenopus homologues of V1 neurons may facilitate fast swimming movements, in much the same way that their mammalian counterparts are required for fast 'walking' movements.

The exact contribution individual V1 interneuron subtypes make to regulating the duration of motor neuron bursting during fictive locomotion remains to be determined. Inhibition of Renshaw cells with cholinergic blockers causes a small increase in the step-cycle period $^{26}$ (S.G. and O.K., unpublished observations), suggesting that a group of non-Renshaw V1 neurons or the V1 population as a whole regulates the step-cycle period during fictive locomotion. Although Renshaw cells and Ia inhibitory interneurons have described roles in neurons. d, Recording from a V1 neuron expressing AlstR in a P1 $E n 1^{\mathrm{Cre}}$; AlstR192; $Z n G$ mouse in response to current steps. e, Recordings from an AlstR-expressing V1 neuron in response to a current ramp. Before allatostatin $(\mathrm{AL})$ application, the cell begins to fire upon current injection of $105 \mathrm{pA}$. After allatostatin application $(10 \mathrm{nM})$, the cell only fires when the injected current reaches $190 \mathrm{pA}$. f, Electroneurogram recordings from IL2 and IL5 ventral roots in a P1 En1 ${ }^{\mathrm{Cre}}$; AlstR192 mouse, showing the effects of allatostatin $(5 \mu \mathrm{M})$ application and wash-out on locomotor activity in the isolated spinal cord. The lengthening of the step cycle after allatostatin application was significant $(P=0.02$, paired $t$-test; $n=15$ spinal cords).

coordinating flexor-extensor motor neurons during spinal reflexes ${ }^{27}$, our study demonstrates that the V1 population as a whole does not have a primary role in regulating locomotor CPG flexor-extensor activity. It therefore seems that an additional group of non-V1 interneurons have been incorporated into the walking CPG of terrestrial vertebrates to secure flexor-extensor alternation.

Our study also demonstrates that the acute silencing of a select population of neurons using genetic approaches can be used to elucidate their function with respect to a defined behaviour such as locomotion. Our results highlight the feasibility of using ligandactivated GIRK channels to selectively manipulate neuronal excitability in the vertebrate nervous system, and the conditional AlstR192 mice described here can be used to selectively silence neurons throughout the nervous system. This and similar genetic approaches now make it feasible to selectively probe the function of small populations of neurons, which should facilitate the mapping of neural circuits at higher resolutions than was previously possible.

\section{METHODS}

Animals. The generation and genotyping of the Pax6 $6^{\text {lacZ }}, S e y, E n 1^{\mathrm{Cre}}, E n 1^{t l Z}$, $W n t 1^{\text {En1 }}$, nestin ${ }^{\text {Cre }}, R 26$-lac $Z^{\text {flox }} / D T A, R 26^{\text {lacZ }}$ and $R 26^{\text {GFP }}$ alleles in mice has been previously described ${ }^{8,9,12,23,28}$. Embryos and tissues were obtained from timed matings.

Generation of conditional AlstR mice. The Drosophila AlstR coding sequence, followed by an IRES-EGFP ${ }^{24}$, was inserted downstream of a loxP-flanked $\beta$ geo/ polyA stop sequence in the conditional $Z / E G$ construct ${ }^{29}$ to produce the $Z / A l s t R$ expression vector (see Fig. 4a). The linearized Z/AlstR construct was electroporated into the embryonic stem cell line W9.5. G418-resistant clones were screened for uniform and robust lacZ expression, and for single transgene integrants. Two clones gave germline transmission (AlstR172 and AlstR192). 
AlstR192 mice that showed lacZ expression throughout the nervous system were used for all further experiments.

In situ hybridization and immunohistochemistry. Immunostainings were performed as previously described ${ }^{5}$. Images were captured using a Zeiss LSM510 confocal microscope and assembled using Adobe Photoshop. Cell numbers are indicated as mean \pm s.d. per section per spinal cord side, and were determined on counts of at least three embryos (five sections each). In situ hybridization and staining for $\beta$-galactosidase activity were performed as previously described ${ }^{5,8}$.

Electrophysiology: electroneurogram recordings. Electrophysiological experiments were performed on E18.5-P2 mice in accordance with the ethical rules stipulated by NIH and the Swedish government. Animals were anaesthetized and decapitated, and spinal cords were dissected out in ice-cold Ringers' solution ${ }^{4}$. Recordings were made in Ringers' solution at $20^{\circ} \mathrm{C}$ by placing bipolar suction electrodes on three of the second and fifth lumbar ventral roots (that is rL2, 1L2, rL5, lL5). Electroneurogram signals were amplified, band-pass filtered $(100 \mathrm{~Hz}-1 \mathrm{kHz})$, digitized and collected using Axoscope software (Axon Instruments). Rhythmic locomotor activity was induced by adding NMDA $(5 \mu \mathrm{M})$ and 5 -HT $(5-30 \mu \mathrm{M})$ to the Ringers' solution. Effects of allatostatin $(10 \mathrm{nM}-5 \mu \mathrm{M})$ were examined by adding the peptide to the perfusion solution.

Electrophysiology: analysis of locomotor activity. Step-cycle period and burst duration were determined by analysing lL2 or rL2 activity ${ }^{4}$. Step-cycle period and burst duration averages were determined from all recorded bursts after the onset of stable locomotor-like activity. The effects of allatostatin were measured 10 min after application to allow for drug wash-in. All measurements are given \pm s.d. $P$ values less than 0.05 were considered significant. Circular statistics ${ }^{19}$ were used to determine the coupling strength between left and right ventral roots, as well as between flexor-related (L2) and extensor-related (L5) ventral roots on the same side of the spinal cord. L2 bursts were used as a reference. Vector points representing the phase values between 0 and 1 were plotted for each experiment, and show the mean phase as well as the concentration of phase values around the mean. Appropriate left-right or flexor-extensor alternation is represented by phase values around 0.5 .

Electrophysiology: intracellular recordings. Whole-cell patch-clamp recordings from motor neurons were made using recording pipettes with a resistance of 4-5 M . The microelectrode was driven into the spinal cord through a pial patch in the ventrolateral surface. Cells were patched in the motor neuron area and recorded using an AxoClamp 2B amplifier (Axon Instruments).

Electrophysiology: acute spinal cord slice recordings. For slice recordings, spinal cord slices $250-300-\mu \mathrm{m}$ thick were cut using a Leica VY1000E vibrating microtome. After a 1-h recovery period, slices were transferred to a recording chamber, mounted on an Olympus BX51W1 microscope and perfused with oxygenated Ringers' solution at room temperature. GFP-positive cells were visualized using a DAGE-MTI IR-1000 CCD camera and patched visually using a Sutter MPC-325 micromanipulator. Recordings were made in currentclamp mode using a MultiClamp 700B amplifier (Axon Instruments).

Behavioural testing. Rotarod test were performed during the light phase of a $12 \mathrm{~h}: 12 \mathrm{~h}$ light:dark cycle as previously described ${ }^{30}$. Mice were placed on a Rotamex 4/8 (Columbus Instruments) idling at 5 r.p.m. Rotarod speed was set to increase gradually from 5 to 65 r.p.m. over the course of $3 \mathrm{~min}$. Retention time on the rod was recorded for three trials per mouse per day for five consecutive days, using three mutant and three wild-type mice.

\section{Received 15 November; accepted 22 December 2005.}

1. Grillner, S. The motor infrastructure: from ion channels to neuronal networks. Nature Rev. Neurosci. 4, 573-586 (2003).

2. Kiehn, O. \& Kjaerulff, O. Distribution of central pattern generators for rhythmic motor outputs in the spinal cord of limbed vertebrates. Ann. NY Acad. Sci. 860, 110-129 (1998)

3. Roberts, A., Soffe, S. R., Wolf, E. S., Yoshida, M. \& Zhao, F. Y. Central circuits controlling locomotion in young frog tadpoles. Ann. NY Acad. Sci. 860, 19-34 (1998).

4. Butt, S. J., Harris-Warrick, R. M. \& Kiehn, O. Firing properties of identified interneuron populations in the mammalian hindlimb central pattern generator. J. Neurosci. 22, 9961-9971 (2002).

5. Lanuza, G. M., Gosgnach, S., Pierani, A., Jessell, T. M. \& Goulding, M. Genetic identification of spinal interneurons that coordinate left-right locomotor activity necessary for walking movements. Neuron 42, 375-386 (2004).

6. Angel, M. J., Jankowska, E. \& McCrea, D. A. Candidate interneurones mediating group I disynaptic EPSPs in extensor motoneurones during fictive locomotion in the cat. J. Physiol. (Lond.) 563, 597-610 (2005).

7. Jessell, T. M. Neuronal specification in the spinal cord: inductive signals and transcriptional codes. Nature Rev. Genet. 1, 20-29 (2000).
8. Saueressig, H., Burrill, J. \& Goulding, M. Engrailed-1 and netrin-1 regulate axon pathfinding by association interneurons that project to motor neurons. Development 126, 4201-4212 (1999).

9. Sapir, T. et al. Pax6 and Engrailed-1 regulate two distinct aspects of Renshaw cell development. J. Neurosci. 24, 1255-1264 (2004)

10. Li, W. C., Higashijima, S., Parry, D. M., Roberts, A. \& Soffe, S. R. Primitive roles for inhibitory interneurons in developing frog spinal cord. J. Neurosci. 24, 5840-5848 (2004).

11. Higashijima, S., Masino, M. A., Mandel, G. \& Fetcho, J. R. Engrailed-1 expression marks a primitive class of inhibitory spinal interneuron. J. Neurosci. 24, 5827-5839 (2004)

12. Brockschnieder, D. et al. Cell depletion due to diphtheria toxin fragment A after Cre-mediated recombination. Mol. Cell. Biol. 24, 7636-7642 (2004).

13. Ericson, J. et al. Pax6 controls progenitor cell identity and neuronal fate in response to graded Shh signaling. Cell 90, 169-180 (1997).

14. Jiang, Z., Carlin, K. P. \& Brownstone, R. M. An in vitro functionally mature mouse spinal cord preparation for the study of spinal motor networks. Brain Res. 816, 493-499 (1999).

15. Kullander, K. et al. Role of EphA4 and EphrinB3 in local neuronal circuits that control walking. Science 299, 1889-1892 (2003).

16. Wenner, P., O'Donovan, M. J. \& Matise, M. P. Topographical and physiological characterization of interneurons that express engrailed-1 in the embryonic chick spinal cord. J. Neurophysiol. 84, 2651-2657 (2000).

17. Alvarez, F. J. et al. Postnatal phenotype and localization of V1-derived interneurons. J. Comp. Neurol. 493, 177-192 (2005).

18. Jankowska, E., Fu, T. C. \& Lundberg, A. Reciprocal la inhibition during the late reflexes evoked from the flexor reflex afferents after DOPA. Brain Res. 85 , 99-102 (1975).

19. Kjaerulff, O. \& Kiehn, O. Distribution of networks generating and coordinating locomotor activity in the neonatal rat spinal cord in vitro: a lesion study. J. Neurosci. 16, 5777-5794 (1996).

20. Kiehn, O., Hounsgaard, J. \& Sillar, K. T. in Neurons, Networks, and Motor Behaviour (eds Stein, P. S. G., Stuart, D., Selverston, A. \& Grillner, S.) 47-59 (MIT Press, Cambridge, Massachusetts, 1997).

21. Kjaerulff, O. \& Kiehn, O. Crossed rhythmic synaptic input to motoneurons during selective activation of the contralateral spinal locomotor network. J. Neurosci. 17, 9433-9447 (1997).

22. Butt, S. J. \& Kiehn, O. Functional identification of interneurons responsible for left-right coordination of hindlimbs in mammals. Neuron 38, 953-963 (2003).

23. Danielian, P. S. \& McMahon, A. P. Engrailed-1 as a target of the Wnt-1 signalling pathway in vertebrate midbrain development. Nature 383, 332-334 (1996).

24. Lechner, H. A., Lein, E. S. \& Callaway, E. M. A genetic method for selective and quickly reversible silencing of mammalian neurons. J. Neurosci. 22, 5287-5290 (2002).

25. Borodinsky, L. N. et al. Activity-dependent homeostatic specification of transmitter expression in embryonic neurons. Nature 429, 523-530 (2004).

26. Myers, C. P. et al. Cholinergic input is required during embryonic development to mediate proper assembly of spinal locomotor circuits. Neuron $46,37-49$ (2005).

27. Burke, R. E. in The Synaptic Organization of the Brain (ed. Shepherd, G. M.) 77-120 (Oxford Univ. Press, Oxford, 1998).

28. St-Onge, L., Sosa-Pineda, B., Chowdhury, K., Mansouri, A. \& Gruss, P. Pax6 is required for differentiation of glucagon-producing $\alpha$-cells in mouse pancreas. Nature 387, 406-409 (1997).

29. Novak, A., Guo, C., Yang, W., Nagy, A. \& Lobe, C. G. Z/EG, a double reporter mouse line that expresses enhanced green fluorescent protein upon cre-mediated excision. Genesis 28, 147-155 (2000).

30. Brandon, E. P. et al. Choline transporter 1 maintains cholinergic function in choline acetyltransferase haploinsufficiency. J. Neurosci. 24, 5459-5466 (2004).

Supplementary Information is linked to the online version of the paper at www.nature.com/nature.

Acknowledgements We thank J. Rivier for the allatostatin peptide, P. Gruss and A. Mansouri for Pax6 mutant mice, P. Soriano for Rosa26 lacz mice, A. McMahon for $W_{n t 1}{ }^{E n 1}$ mice, S. Narayan for generating ZnG reporter mice, and M. Gross, A. Joyner, S. Pfaff and P. Slesinger for materials. We thank G. Lemke, S. Pfaff, P. Gray and K. Quinlan for their comments on the manuscript. This research was supported by grants from the National Institutes of Health (M.G., O.K.) and the Human Frontiers Science Program (O.K. and M.G.). G.M.L. was supported by an HFSP postdoctoral fellowship.

Author Contributions are provided in the Supplementary Information.

Author Information Reprints and permissions information is available at npg.nature.com/reprintsandpermissions. The authors declare no competing financial interests. Correspondence and requests for materials should be addressed to M.G. (goulding@salk.edu). 